\title{
Synthesis of luminescent silicon clusters by spark ablation
}

\author{
Winston A. Saunders, ${ }^{\text {a) }}$ Peter C. Sercel, ${ }^{\text {b) }}$ R. B. Lee, Harry A. Atwater, \\ Kerry J. Vahala, Richard C. Flagan, and Ernesto J. Escorcia-Aparcio ${ }^{\text {c) }}$ \\ California Institute of Technology, Pasadena, California 91125
}

(Received 26 February 1993; accepted for publication 8 July 1993)

\begin{abstract}
The synthesis of luminescent nanometer-scale Si clusters by spark ablation from a crystalline $\mathrm{Si}$ substrate is described. The cluster source, described in the text, generates clusters in a flowing $\mathrm{Ar}$ stream at atmospheric pressure. Electron microscopy reveals that the clusters have diameters in the 2-4 nm size range. The luminescence spectra of the clusters, similar to that of porous $\mathrm{Si}$, are presented.
\end{abstract}

The recent observation of luminescence from porous$\mathrm{Si}^{1}$ and $\mathrm{Si}$ clusters ${ }^{2}$ suggests that under appropriate conditions nanometer-scale Si can produce visible luminescence. While many details of the microstructure of porous Si remain unclear, wirelike, ${ }^{3}$ dotlike, ${ }^{4}$ and amorphous ${ }^{5}$ structures have been proposed. In comparison, several techniques have been demonstrated by which nanometer-scale Si clusters produce visible luminescence. These include direct formation of clusters by gas-phase decomposition of silane $e^{2,6}$ and the formation of clusters by atomic diffusion in glass matrices. ${ }^{7}$ In the case of clusters, morphology questions are more directly resolved, owing to the simpler structure of the samples in question. Thus, cluster samples have some advantages over (intrinsically more complex) porous silicon regarding the systematic investigation of mechanisms for luminescence.

Here, we report on a method for the generation of luminescent $\mathrm{Si}$ clusters by direct ablation from crystalline Si substrates. The present method, unlike those proposed previously, relies on direct vapor-phase synthesis of nanometer-scale clusters by the vaporization of a bulk sample in an inert atmosphere, thus avoiding many contamination problems which may exist in synthesis from hydride sources. The discussion in the first part of this letter focuses on the cluster source, which uses a high energy electric spark to ablate crystalline Si electrodes. In the second part of the letter we discuss characterization of the clusters, including transmission electron micrographs and optical measurements.

The clusters are formed using a continuous-flow sparkablation (spark-) source, shown schematically in Fig. 1. The spark-source vaporizes material directly from the crystalline Si electrodes using a high energy electric spark. The vapor subsequently nucleates in a flowing Ar jet to form clusters. The carrier gas (Matheson, 99.9995\%) is taken directly from the cylinder without further purification. The source body is constructed of a single piece of Delrin, drilled to accommodate two $\frac{3}{8}$ in. diam stainless steel electrodes and a $\frac{1}{8}$ in. gas inlet. The electrodes are fitted with screw end caps which tighten an o-ring seal between the

\footnotetext{
a) Present address: Intel Corporation, 2200 Mission College Blvd., Santa Clara, CA 95052.

b) Present address: Physics Department, University of Oregon, Eugene, OR 97401.

${ }^{c}$ Present address: Physics Department, University of California, Berkeley, CA 94720.
}

electrodes and the source body while the gas inlet is pressfit into $a \frac{1}{8}$ in. hole. The source is mounted onto a vacuum chamber which is exhausted by a zeolyte-trapped mechanical pump.

A flashlamp circuit iš used to drive the spark source. Trial and error has shown that a spark energy between 50 and $150 \mathrm{~mJ}$ is optimal for the production of clusters in the desired size range. Significantly lower spark energy vaporizes little material and consequently cluster production is poor. Significantly higher energies tend to shatter the electrodes, presumably as a result of mechanical and/or acoustic shock from the resulting spark. It is interesting to note that the energy of the spark is comparable in magnitude to the (laser pulse) energy used in laser vaporization cluster sources. The components of the pulser circuit are chosen to give a pulse width of $2 \mu \mathrm{s}$ and a repetition rate of about 200 $\mathrm{Hz}$. Under normal conditions, the flow rate through the Ar jet is about 1 standard liter per minute. The pressure of the chamber, between 0.6 and $1.2 \mathrm{~atm}$, is regulated by adjusting a needle valve between the chamber and the mechanical pump.

Clusters generated by the source are carried downstream by the flowing Ar and are collected on a suitable substrate. Typically, $\langle 100\rangle$ single-crystal Si substrates are used, though other substrates (e.g., graphite and $\mathrm{NaCl}$ ) have been employed, depending on the characterization method employed. We observe that a bias potential applied to the substrate does not significantly affect the surface coverage by the clusters, suggesting that the particles generated by the source are largely uncharged or else have

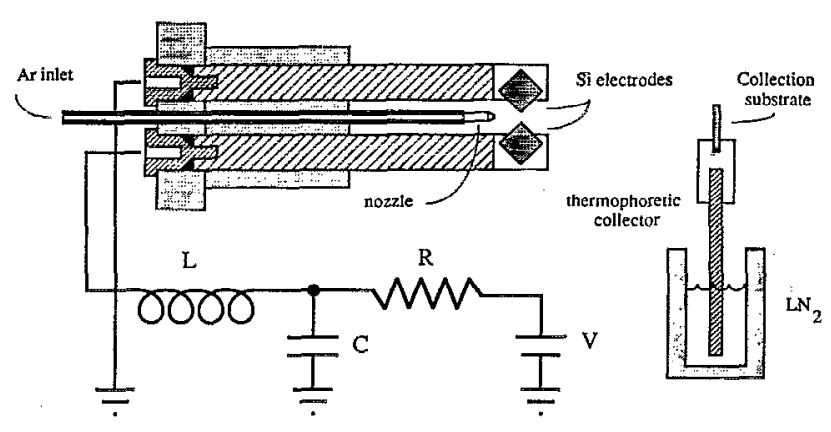

FIG. 1. A schematic diagram of the spark source used to generate luminescent nanometer-scale clusters. A flashlamp driver circuit is utilized to extract the energy stored in the capacitor. Typically, the spark duration is $2 \mu s$ and the spark energy is about $100 \mathrm{~mJ}$. 

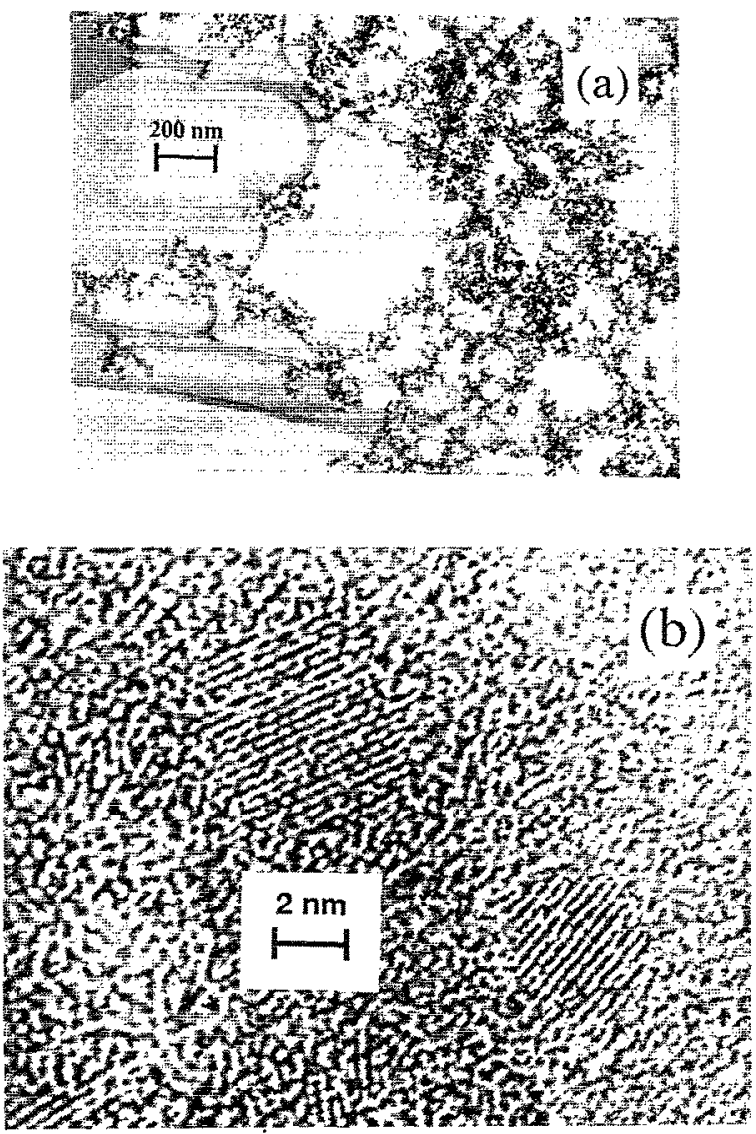

FIG. 2. (a) A low resolution electron micrograph of Si clusters produced by the spark source. Owing to the high density of clusters produced by the source, the individual clusters agglomerate into long, fractal-like chains. The chains are supported on a holey carbon substrate. (b) A high resolution electron micrograph of a section of one chain. Among the many randomly oriented clusters in the field of view, two individual clusters are oriented such that lattice planes are visible. The smaller cluster is approximately 15 atomic rows across.

been neutralized by the time they are collected. In a typical 20 min run, film thicknesses equivalent to a $10 \mathrm{~nm}$ continuous film are produced, as revealed by Rutherford backscattering measurements.

Both a high-resolution and low-resolution transmission electron micrograph of the collected particles is shown in Fig. 2. Owing to the high density of clusters produced by the source, agglomerated chains containing up to several thousand individual clusters, such as shown in the low resolution micrograph, are observed. These agglomerates are formed in the gas phase, prior to collection on the substrate. At higher magnification it is revealed that the agglomerated chains are composed of individual nanometer-scale crystallites. In the high resolution micrograph of Fig. 2(b), two particles with [110] zone axes close to the beam direction, thus revealing lattice fringes, are apparent. Since the particles in the agglomerate are randomly oriented, only a small fraction satisfy this condition. The smaller of the two particles has approximately 15 lattice planes. Electron diffraction shows that the clusters have a bulk-like diamond cubic crystal structure. In general, we observe that samples which contain a higher density of crystallites exhibit stronger luminescence.

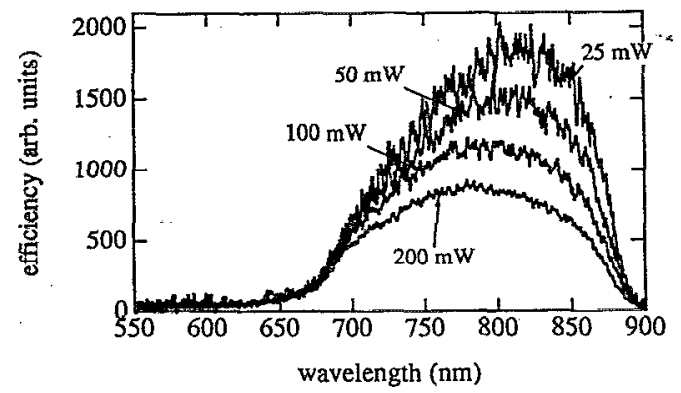

FIG. 3. The relative luminescence efficiency (luminescence intensity normalized to the pump laser power) of a Si cluster sample for various laser powers. The spectra are not corrected for the photomultiplier tube responsie or the grating efficiency. The luminescence efficiency depends weakly on power below about $25 \mathrm{~mW}$.

Luminescence spectra produced by varying excitation power at $488 \mathrm{~nm}$ are shown in Fig. 3. The measurements were performed in air on samples that have undergone aging in air for several days (see below). The luminescence intensities are normalized to the pump laser power. In general, the luminescence intensity rises above $700 \mathrm{~nm}$. The spectra are corrected neither for the response of the GaAs photomultiplier tube nor the monochromator grating efficiency. Roll off of the photomultiplier tube photocathode response is responsible for the decrease in the detected luminescence intensity beyond $850 \mathrm{~nm}$. It is reasonable to expect that the luminescence extends beyond $900 \mathrm{~nm}$, as do other Si cluster luminescence spectra., ${ }^{2,6}$

The cluster luminescence efficiency decreases for powers above about $25 \mathrm{~mW}$. Preliminary time-resolved luminescence decay measurements give decay constants for the clusters in the range of $5 \times 10^{5} \mathrm{~s}^{-1}$. This is comparable to the decay rates for porous $\mathrm{Si}^{8}$ The observed power saturation may be lifetime related due to cluster heating effects.

Immediately upon removal from the vacuum chamber, the cluster samples generally show little or no visible photoluminescence. However, after several minutes exposure to air the luminescence of the particles improves dramatically. This is in contrast to the case of porous $\mathrm{Si}$, which is found to degrade over time, ${ }^{9}$ but similar to the behavior observed for spark-erosion generated porous $\mathrm{Si}^{10}$ The increase in the luminescence intensity continues for approximately $6 \mathrm{~h}$, beyond which little or no change is observed. Samples aged for several weeks, on the other hand, show little or no degradation in their luminescence efficiency. The increase of the luminescence efficiency upon exposure to air suggests that the chemical composition of the cluster surface plays an important role in determining the luminescence efficiency of the clusters.

We have found that the increase in luminescence efficiency is accelerated by laser irradiation. This is shown in Fig. 4, where the luminescence intensity, measured at 800 $\mathrm{nm}$, is plotted as a function of time. The laser power was $100 \mathrm{~mW}$ over a spot size of approximately $3 \mathrm{~mm}^{2}$ at a wavelength of $488 \mathrm{~nm}$. The luminescence intensity rises linearly with time, except for periods where the laser is blocked, during which there is no perceptible change in the 


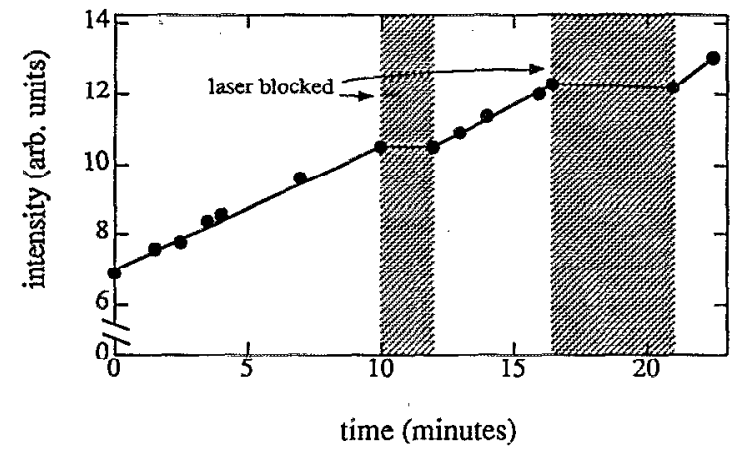

FIG. 4. The luminescence intensity of a cluster sample at $800 \mathrm{~nm}$ as a function of time. The clusters are being pumped at $488 \mathrm{~nm}$ with $100 \mathrm{~mW}$ in a spot size of approximately $3 \mathrm{~mm}^{2}$. During periods where the laser is blocked, the luminescence efficiency is constant. Since laser heating effects are not expected to be important at these power levels, it is likely that photo-generated carriers are accelerating the passivation reaction at the cluster surface.

efficiency. Since laser heating is not expected to be important at these power levels, it is likely that photogenerated carriers play a role in accelerating the increase in luminescence efficiency.

Rutherford backscattering measurements using $2 \mathrm{MeV}$ ${ }^{4} \mathrm{He}^{+}$ions on a luminescent cluster sample mounted on a carbon substrate give a ratio of oxygen to $\mathrm{Si}$ atoms of 0.82 \pm 0.04 . This observation is consistent with a uniform sample of $4 \mathrm{~nm}$ diam Si clusters surrounded by a $1 \mathrm{~nm}$ thick $\mathrm{SiO}_{2}$ layer. However, we cannot rule out the presence of hydroxide groups as hydrogen concentration cannot be measured in the conventional backscattering geometry. In addition, studies have shown that the native oxide on nanometer-scale $\mathrm{Si}$ particles is closer to $\mathrm{SiO}_{1.2},{ }^{11}$ which would modify the conclusions drawn here. We are currently investigating in detail the role which oxygen and water vapor play in passivating the cluster surface.

We have presented results on the generation of lumi- nescent Si clusters using a spark discharge source. The source is simple to construct, robust, and, under appropriate conditions, efficiently produces crystalline nanometerscale Si clusters. The clusters display visible photoluminescence. The observed luminescence from the cluster samples extends in a broad band from approximately $700 \mathrm{~nm}$ to beyond $900 \mathrm{~nm}$, similar, though not identical, to reported spectra of porous $\mathrm{Si}^{1}{ }^{1}$ On the other hand, the spectrum is quite similar to the spectra of $\mathrm{Si}$ clusters produced by the decomposition of silane ${ }^{2}$ as well as those embedded in $\mathrm{SiO}_{2} .{ }^{7}$ This suggests that the luminescence in these systems share a common origin. On the basis of these measurements, it is impossible to say whether the origin of the observed luminescence is due to a quantum size effect. However, given the similarity between the spectra of samples whose main commonality is the presence of nanometer-scale $\mathrm{Si}$ crystallites, it is reasonable to assert that the clusters are responsible for the luminescence, whatever its origin.

We acknowledge support by the NSF Contract No. CTS-8912328. P.C.S. acknowledges support by AT\&T.

${ }^{1}$ L. T. Canham, Appl. Phys. Lett. 57, 1046 (1990).

${ }^{2}$ H. Takagi, H. Ogawa, Y. Yamazaki, A. Ishizaki, and T. Nakagiri, Appl. Phys. Lett. 56, 2379 (1990).

${ }^{3}$ A. G. Cullis and L. T. Canham, Nature 353, 335 (1991).

${ }^{4}$ M. W. Cole, J. F. Harvey, R. A. Lux, D. W. Ekart, and R. Tsu, Appl. Phys. Lett. 60, 2800 (1992).

${ }^{5}$ T. George, M. S. Anderson, W. T. Pike, T. L. Lin, R. W. Fathauer, K. H. Jung, and D. L. Kwong, Appl. Phys. Lett. 60, 2359 (1992).

${ }^{6}$ K. A. Littau, P. J. Szajowski, A. J. Muller, A. R. Kortan, and L. Brus, J. Phys. Chem. 97, 1224 (1993).

${ }^{7}$ M. Fujii, S. Hayashi, and K. Yamamoto, Jpn. J. Appl. Phys. 30, 687 (1991).

${ }^{8}$ G. W. 't Hooft, Y. A. R. R. Kessener, G. L. J. A. Rikken, and A. H. J. Venhuizen, Appl. Phys. Lett. 61, 2344 (1992).

${ }^{9}$ J. N. Kidder, Jr., P. S. Williams, T. P. Pearall, D. T. Schwarz, and B. Z. Nosho, Appl. Phys. Lett. 61, 2869 (1992).

${ }^{10}$ R. E. Iummel and S. Chang, Appl. Phys. Lett. 61, 1965 (1992).

"S. Hayashi, S. Tanimoto, and K. Yamamoto, J. Appl. Phys. 68, 5300 (1990). 